Check for updates

Cite this: RSC Adv., 2017, 7, 40791

\title{
Thermal-sensitive magnetic nanoparticles for dual- modal tumor imaging and therapy
}

\author{
Yan $\mathrm{Xu}^{\mathrm{a}}{ }^{\mathrm{C}}$ Chengcheng Niu, (D) ${ }^{* \mathrm{~b}}$ Senbo An, ${ }^{\mathrm{c}}$ Shixiong Tang, ${ }^{\mathrm{d}}$ Ping Xiao, ${ }^{\mathrm{a}}$ Qinghai Peng ${ }^{\mathrm{b}}$ \\ and Long Wang ${ }^{\mathrm{C}}$
}

"Nanotheranostics" has attracted much attention due to the development of nanomaterials with integrated diagnostic and therapeutic functions. In this work, we fabricated a thermal-sensitive nanotheranostic agent, phase-shifted magnetic nanoparticles (PMNPs), which effectively absorbs heat to achieve hyperthermic therapy in response to NIR irradiation, synergistically enhancing tumor therapy via a phase-shift thermoelastic expansion effect, and can be used as a dual-modal contrast agent for US/MR imagingguided hyperthermia therapy. Histology and electron microscopy confirmed severe cell damage injuries, with fragmentation of lysed cells and nuclear membranes, leaving a mass of vacuoles, and many microbubbles generated with $\mathrm{Fe}_{3} \mathrm{O}_{4}$ nanoparticles expelled to the edge or outside of the microbubbles, thereby achieving a high concentration dose at the target tissue and minimizing systemic side effects.

Received 24th June 2017

Accepted 16th August 2017

DOI: $10.1039 / \mathrm{c} 7 \mathrm{ra07024a}$

rsc.li/rsc-advances

associated with clinical efficacy, disease-free survival, and long-

\section{Introduction}

Commonly used clinical chemotherapy has many limitations, such as undesired side effects induced by nonspecific drug delivery and limited therapeutic efficacy resulting from drug resistance after a period of treatment. ${ }^{1}$ Combination therapy is considered a promising strategy to minimize side effects, improve therapeutic efficiency and overcome drug resistance., ${ }^{2,3}$ For example, chemotherapy can be combined with other therapies, such as photothermal therapy, photodynamic therapy, hyperthermia therapy and radiation therapy to kill cancer cells via a synergistic effect. ${ }^{4-7}$

Hyperthermia therapy (HTT) employs localized thermal energy to "burn" cancer cells that results in cell protein denaturation and coagulation necrosis. ${ }^{\mathbf{8}, 9}$ However, although clinical use of hyperthermia processes is still controversial, HTT is

${ }^{a}$ Department of Renal Medicine, Xiangya Hospital, Central South University, Changsha, Hunan 410008, China

${ }^{b}$ Department of Ultrasound Diagnosis, The Second Xiangya Hospital, Central South University, Changsha, Hunan 410011, China. E-mail: cici204675@163.com

'Department of Orthopedics, Xiangya Hospital, Central South University, Changsha, Hunan 410008, China

${ }^{d}$ Department of Radiology, The Second Xiangya Hospital, Central South University, Changsha, Hunan 410011, China

Chengcheng Niu is a doctor at the second Xiangya hospital, Central South University. She received her BS degrees in medical imaging from Chongqing Medical University in 2008 and her PhD degree in Medical imaging and nuclear medicine from the Institute of Ultrasound imaging in 2013. Her current research interests include ultrasound microbubbles, drug delivery, and multimodal medical imaging. term local recurrence rates. ${ }^{\mathbf{1 0}}$ Among the various types of combination therapies, combined hyperthermia and chemotherapy could trigger drug release from drug carriers inside cells, enhance drug uptake by cells, or enhance antitumor efficacy via a synergistic effect. ${ }^{\mathbf{4 , 1 1}}$

Recently, "nanotheranostics" has attracted much attention due to the development of nanomaterials with integrated diagnostic and therapeutic functions. ${ }^{12-16}$ These nanomaterials can simultaneously serve as multifunctional contrast agents, such as for magnetic resonance (MR), ultrasound (US) or fluorescence imaging of tumors and drug delivery or ablation of tumors. However, the use of inorganic nanomaterials with a non-biodegradable nature has significantly hampered clinical use of nanotheranostic agents. Therefore, there is an urgent need to design biocompatible and biodegradable nanomaterials that can smartly incorporate therapeutic and diagnostic functions for combination cancer therapy.

Poly(lactide-co-glycolide) (PLGA), approved by the U.S. Food and Drug Administration (FDA), is one of the most widely used polymers due to its biodegradability and biocompatibility. Previously, we developed PLGA microbubbles carrying indocyanine green (ICG), $\mathrm{Fe}_{3} \mathrm{O}_{4}$ nanoparticles or doxorubicin for US, MR or fluorescence imaging and drug delivery for antitumor therapy. ${ }^{17-19} \mathrm{Fe}_{3} \mathrm{O}_{4}$ nanoparticles have been widely used in biomedical research for MR imaging and HTT., ${ }^{\mathbf{4 2 0 , 2 1}}$ Espinosa et al. reported a high efficacy of $\mathrm{Fe}_{3} \mathrm{O}_{4}$ nanocubes in dual-mode hyperthermia (alternating magnetic field and laser hyperthermia). ${ }^{20}$ Hayashi et al. reported smart nanoparticles that contained clustered $\mathrm{Fe}_{3} \mathrm{O}_{4}$ nanoparticles and doxorubicin and could generate heat in response to an alternating current magnetic field and subsequently release doxorubicin. ${ }^{4}$ 


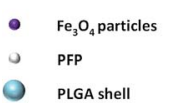

Fig. 1 Schematic illustration of the structure of PMNPs and a schematic of the phase-transition process via NIR-induced vaporization.

Recent studies have shown that ultrasound contrast agents that encapsulate perfluorocarbon (PFC) liquid droplets produce excellent ultrasound imaging contrast by transforming to gas bubbles via acoustic or optical activated vaporization. ${ }^{22-25}$ Furthermore, this vaporization property has been gainfully employed for thermal ablation of tumors ${ }^{24-27}$ and to deliver encapsulated chemotherapeutic agents to a target region. ${ }^{\mathbf{2 8 - 3 0}}$ However, to our knowledge, there have been few reports regarding the process of drug delivery from the gas bubbles formed under vaporization. In our previous study, we used the dye DiI as a drug model to observe the drug release process after vaporization in vitro. ${ }^{31}$ In this work, $\mathrm{Fe}_{3} \mathrm{O}_{4}$ nanoparticles as a payload on the nanoparticles in tumor tissues were observed by TEM to further investigated their release after vaporization. The progress of $\mathrm{Fe}_{3} \mathrm{O}_{4}$ nanoparticles release after vaporization may be useful for the understanding of drug release from an inflated bubble.

Herein, we developed temperature-responsive polymeric phase-shifted magnetic nanoparticles (PMNPs) that can be used as a dual-modal contrast agent for US/MR imaging-guided therapy and effectively absorb heat by NIR irradiation, enhancing tumor therapy via a phase-shift expansion. The PMNPs comprised two parts: (1) the polymer shells are composed of biocompatible PLGA; (2) the liquid core is composed of perfluoropentane (PFP) with a boiling point of approximately $29^{\circ} \mathrm{C}$. The PFP nanodroplets easily convert into nano and/or microbubbles upon heating to physiological temperatures. $^{30,32}$ Schematic illustration of the structure of PMNPs and the phase-transition process is shown in Fig. 1.

\section{Materials and methods}

\subsection{Materials}

PLGA (lactide : glycolide $=50: 50, M_{\mathrm{w}}=10000 \mathrm{Da}$ ) was purchased from Shandong Daigang Medical Polymer Materials. Perfluoropentane (PFP) was obtained from Alfa Aesar (UK). Oleic acid-treated $\mathrm{Fe}_{3} \mathrm{O}_{4}$ nanoparticles with a mean diameter of $10 \mathrm{~nm}$ were obtained from Ocean Nano Tech Inc. (USA). Animals were performed in accordance with the guidelines of the Department of Laboratory Animals, Central South University, China, and approved by the Ethics Committee of Central South University.

\subsection{Preparation of PMNPs}

The PMNPs were fabricated using a published method with minor modifications. In a typical procedure, $0.4 \mathrm{~mL}$ of $\mathrm{Fe}_{3} \mathrm{O}_{4}$ particle suspension ( $31 \mathrm{mg}$ Fe per $\mathrm{mL}$ ) and $100 \mathrm{mg}$ of PLGA was added to $2 \mathrm{~mL}$ of $\mathrm{CHCl}_{3}$. Subsequently, $0.8 \mathrm{~mL}$ of liquid PFP was added to $\mathrm{CHCl}_{3}$, and the mixture was emulsified using an ultrasonic processor (SONICS \& MATERIALS, Inc., USA) at a power of $130 \mathrm{~W}$ and frequency of $20 \mathrm{kHz}$ for $1 \mathrm{~min}$ in an ice bath. Then, $10 \mathrm{~mL}$ of cold PVA solution $(5 \% \mathrm{w} / \mathrm{v})$ was poured into the mixture and homogenized in an ice bath using a homogenizer (Omni International, USA) for $5 \mathrm{~min}$ at $9500 \mathrm{rpm}$. Then, $20 \mathrm{~mL}$ of deionized (DI) water was added, and the sample was mixed for $2 \mathrm{~h}$. Finally, the PMNPs were centrifuged at $7500 \mathrm{rpm}$ for $5 \mathrm{~min}$ and washed with DI water 3 times. The same procedure was used to prepare the magnetic nanoparticles (MNPs) in the absence of PFP, and phase-shifted nanoparticles (PNPs) in the absence of $\mathrm{Fe}_{3} \mathrm{O}_{4}$. Blank PLGA nanoparticles were fabricated and used as a control.

\subsection{Characterization of PMNPs}

Scanning electron microscopy (SEM, JEOL-7800F, Japan) was used to observe the morphology of PMNPs. Transmission electron microscopy (TEM, Hitachi H-7600, Japan) was applied to confirm the presence of $\mathrm{Fe}_{3} \mathrm{O}_{4}$ particles in PMNPs shells. A dynamic light scattering (DLS) zetasizer instrument (Nano ZS, Malvern Instruments, UK) was employed to determine the size and surface charge of PMNPs. An atomic absorption spectrometry method was used to measure the number of $\mathrm{Fe}_{3} \mathrm{O}_{4}$ particles encapsulated in the nanoparticles with different iron concentrations.

\subsection{Temperature elevation induced by NIR irradiation}

A portable infrared therapy apparatus $\left(1.4 \mathrm{~W} \mathrm{~cm}^{-2}\right.$, Philips, China) with wavelength coverage of $0.75-1.4 \mu \mathrm{m}$ was used to irradiate the nanoparticles in the mold. The temperature profile of PMNPs was monitored under NIR irradiation using an infrared thermometer (Fluke 62 MAX, USA). Aqueous suspensions of PMNPs, MNPs, PNPs, PLGA NPs and saline (as the negative control) in wells of a 6 -well plate ( $2 \mathrm{~mL}$ per well) were irradiated by a NIR apparatus at a distance of $15 \mathrm{~cm}$ for $10 \mathrm{~min}$. The amount of $\mathrm{Fe}_{3} \mathrm{O}_{4}$ was equivalent in PMNPs and MNPs samples $\left(56 \mu \mathrm{g} \mathrm{mL^{-1 }} \mathrm{Fe}\right)$. The temperatures of the solutions were measured at $30 \mathrm{~s}$ intervals.

\subsection{In vitro MRI assessment of PMNPs}

PNPs without iron-content (I) and PMNPs with different iron concentrations (II, $0.1 \mathrm{mmol} \mathrm{L}^{-1}$; III, $0.2 \mathrm{mmol} \mathrm{L}^{-1}$; IV, $0.3 \mathrm{mmol} \mathrm{L}^{-1} ; \mathrm{V}, 0.4 \mathrm{mmol} \mathrm{L}^{-1}$; VI, $0.5 \mathrm{mmol} \mathrm{L}^{-1}$ ) were placed in $1 \mathrm{~cm}$-diameter cell cryotubes and imaged on a BioSpec 7.0 $\mathrm{T}$ MRI scanner (Burker, Switzerland). MR images were obtained using a $T_{2}$ weighted turbo spin echo (repetition time (TR) $=$ $3000 \mathrm{~ms}$, echo time (TE) $=45 \mathrm{~ms}$ ) sequence. All images were acquired at a section thickness of $1 \mathrm{~mm}$ without an intersection gap; flip angle (FA) $=90^{\circ}$. The MRI signal intensity (SI) within the region of interest was measured. 


\subsection{In vitro NIR-induced phase transition for US imaging with PMNPs}

PMNPs and MNPs (without PFP) suspensions were transplanted onto a custom-made agarose mold with holes in the center (diameter $=1 \mathrm{~cm}$, depth $=2 \mathrm{~cm}$ ). A portable infrared therapy apparatus (1.4 W $\mathrm{cm}^{-2}$, Philips, China) with wavelength coverage of $0.75-1.4 \mu \mathrm{m}$ was used to irradiate the nanoparticles in the mold. The temperature of the nanoparticles in the mold was monitored with an infrared thermometer (Fluke 62 MAX; USA). The phase transition of the nanoparticles was monitored under a microscope (Olympus IX71, Japan). Before and after NIR irradiation when the temperature of the nanoparticles increased to $50{ }^{\circ} \mathrm{C}$, ultrasound images were acquired. A 7.5 $\mathrm{MHz}$ US transducer of Esaote Mylab 70 scanner was used for the US study.

\subsection{In vivo $\mathrm{MR} / \mathrm{US}$ imaging}

$\mathrm{BALB} / \mathrm{c}$ nude mice (20 g, 6-8 weeks old) were used for the imaging experiment. To establish the animal tumor model, MCF-7 cells $\left(1 \times 10^{6}\right)$ were administered via subcutaneous injection into the right flank of the nude mice. In vivo $\mathrm{MR} / \mathrm{US}$ imaging of the tumors was initiated four weeks after tumor inoculation, when the tumors reached a size of 7-9 $\mathrm{mm}$.

Twelve nude mice bearing xenograft tumors were inoculated after one month, and used to observe the enhancement effect of PMNPs on MR and contrast enhanced ultrasound (CEUS) imaging via percutaneous injection into the tumor site. All solutions of PMNPs, PNPs and MNPs were at the same concentration of $0.3 \mathrm{mg} \mathrm{mL} \mathrm{m}^{-1}$; the dose for intratumoral injection was $200 \mu \mathrm{L}$ on each occasion.

MR imaging of nude mice was performed on a BioSpec 7.0 T MRI scanner (Burker, Switzerland). PMNPs or PNPs (without $\mathrm{Fe}_{3} \mathrm{O}_{4}$ ) were injected directly into the tumor site. The MR images were obtained using a $T_{2}$ weighted turbo spin echo (repetition time $[\mathrm{TR}]=2500 \mathrm{~ms}$, echo time $[\mathrm{TE}]=35 \mathrm{~ms}$ ) sequence before and after injection. After all images were acquired, the SI within the ROIs was measured (thickness $=1 \mathrm{~mm}$; flip angle $(\mathrm{FA})=90^{\circ}$ ) and the tumor tissue SI before and after injection were compared.

US imaging of nude mice was carried using a Siemens S3000 ultrasound scanner. Before receiving infrared irradiation, the nude mice in each group were injected directly into the tumor site with PMNPs or MNPs (without PFP) and massaged for $1 \mathrm{~min}$. Subsequently, all mice were exposed to NIR irradiation $(\lambda$ $\left.=0.75-1.4 \mu \mathrm{m}, 1.4 \mathrm{~W} \mathrm{~cm}^{-2}\right)$. A probe of the thermocouple thermometer probe (TES 1310, Taiwan) was inserted into the tumor tissue to monitor tumor temperature during irradiation. Before NIR irradiation and at $10 \mathrm{~s}, 1 \mathrm{~min}$ and $4 \mathrm{~min}$ after irradiation, all mice received ultrasonography at the tumor sites.

\subsection{HTT for tumor ablation}

Twenty-four nude mice bearing xenograft tumors were used to demonstrate the NIR-irradiated HTT effect on tumor ablation. The mice were divided into four groups: (I) saline; (II) PMNPs alone (PMNPs); (III) PLGA combined with NIR irradiation (PLGA + NIR); (IV) PMNPs combined with NIR irradiation (PMNPs +
NIR). In groups III-IV, $200 \mu \mathrm{L}$ of PLGA or PMNP solution was injected directly into the tumor site in nude mice prior to NIR irradiation. Groups I (saline) and II (PMNPs) did not receive NIR irradiation. All mice in groups III-IV received NIR irradiation at a distance of $15 \mathrm{~cm}$ for $4 \mathrm{~min}$ twice. We inserted thermocouple thermometer probe (TES 1310, Taiwan) into the tumor tissue to monitor the temperature of the tumor tissue in all groups. The body weights and tumor sizes in each mouse was recorded every 2 days for 14 days. The tumor volume was calculated according to the formula: (tumor width) ${ }^{2} \times$ (tumor length)/2. After therapy, the tumors of the nude mice were excised for histological analysis and TEM.

\subsection{Biological toxicity assessment}

Fifteen healthy female BALB/c nude mice were used for assessing the biological toxicity of PMNPs. Ten mice were intravenously injected with PMNPs $\left(0.2 \mathrm{~mL}, 2 \mathrm{mg} \mathrm{mL}{ }^{-1}\right)$. The other 5 mice were injected with saline as controls. Serum biochemistry assays were carried at 3 and 14 days after injection of PMNPs. Two indicators of hepatic function, alanine aminotransferase (ALT) and aspartate aminotransferase (AST) levels, and two indicators of renal function, urea nitrogen (UREA) and creatinine (CREA) levels, were measured in blood samples. After 14 days, all the mice were sacrificed, the major organs (lung, heart, liver, spleen, and kidney) were harvested and sections were stained with H\&E.

\section{Results and discussion}

\subsection{Structural characterization of PMNPs}

SEM images (Fig. 2a) revealed a smooth and uniform spherical morphology of PMNPs. TEM images of PMNPs before (Fig. 2b) and after (Fig. 2c) NIR irradiation showed the presence of $\mathrm{Fe}_{3} \mathrm{O}_{4}$ particles in the PMNP shells, as indicated by the large number of black particles evenly embedded in the spherical shell. While after NIR irradiation, the nanoparticles expanded to almost twice of the original size, the black particles in the nanoparticles showed unevenly and aggregately distribution. The nanoparticle size distribution determined by DLS was nearly symmetric (Fig. 2d, mean diameter: $294 \mathrm{~nm}$, PDI $=0.012$ ). The nanoparticle surface was negatively charged (Fig. 2e, zeta potential: $-1.47 \mathrm{mV}$ ). The amount of $\mathrm{Fe}_{3} \mathrm{O}_{4}$ particles encapsulated in the nanoparticles measured by atomic absorption spectrometry was $56.45 \pm 1.06 \mu \mathrm{g} \mathrm{mL}^{-1}$.

\subsection{In vitro HTT effect of PMNPs}

To study the effectiveness of using PMNPs in HTT, aqueous suspensions of PMNPs, MNPs, PNPs, PLGA NPs and saline were exposed to NIR irradiation at a distance of $15 \mathrm{~mm}$ with a power density of $1.4 \mathrm{~W} \mathrm{~cm}^{-2}$ for $10 \mathrm{~min}$. Fig. 3 showed the temperature changes in different groups. A rapidly increasing temperature increase was observed in PMNPs and MNPs groups, while a smaller temperature change was observed in PNPs, PLGA NPs and saline groups compared with the other groups. The maximum temperatures achieved in PMNPs, MNPs, PNPs, PLGA NPs and saline were $58.1^{\circ} \mathrm{C}, 56.5^{\circ} \mathrm{C}, 46.0^{\circ} \mathrm{C}, 45.5^{\circ} \mathrm{C}$, and 

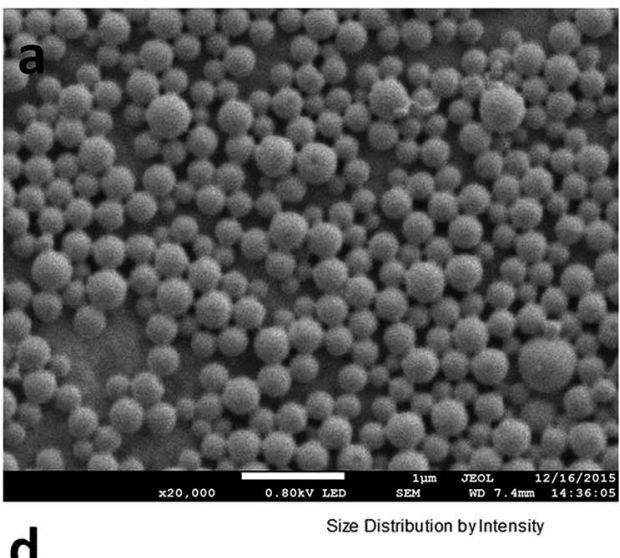

d

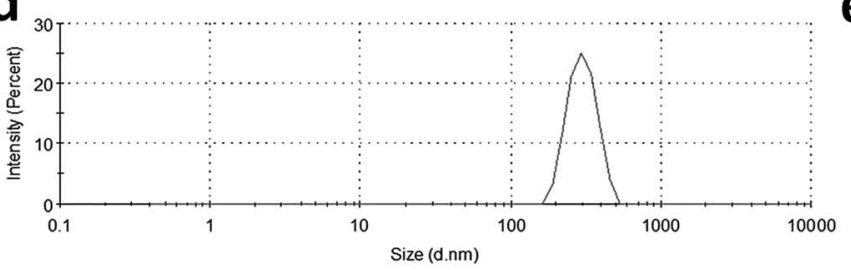

b

$500 \mathrm{~nm}$

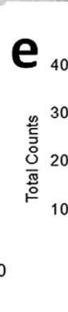

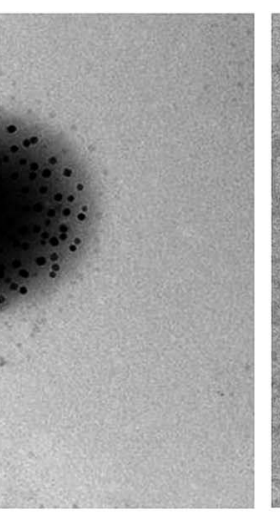

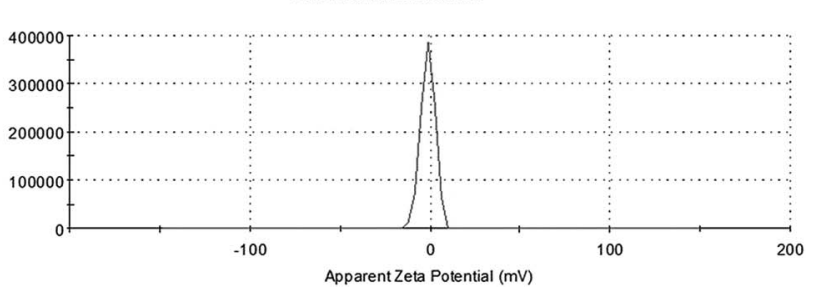

Fig. 2 (a) SEM image of PMNPs (magnification, $20000 \times$; scale bar, $1 \mu \mathrm{m}$ ). TEM images of PMNPs (b) before and (c) after NIR irradiation. (b) A large amount of $\mathrm{Fe}_{3} \mathrm{O}_{4}$ nanoparticles are evenly embedded in the spherical shell in the form of black particles. (c) After NIR irradiation, PMNPs expand and $\mathrm{Fe}_{3} \mathrm{O}_{4}$ nanoparticles are unevenly distributed. (b) Magnification, 37000 ; scale bar, $500 \mathrm{~nm}$. (c) Magnification, $18500 \times$; scale bar, $1 \mu \mathrm{m}$. ( $d$ and e) Size distribution and zeta potential of PMNPs.

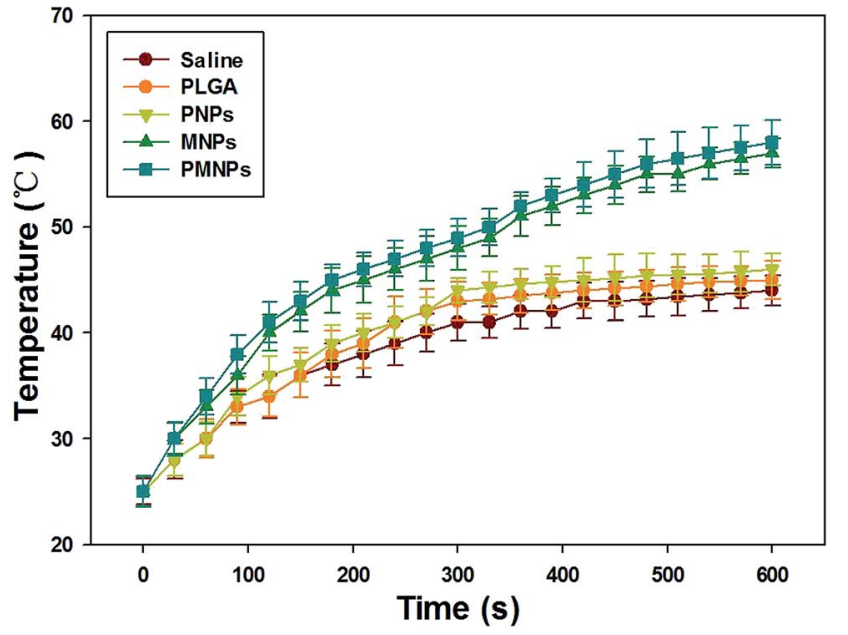

Fig. 3 Temperatures elevation of PMNPs, MNPs, PNPs, PLGA NPs and saline with continuous NIR irradiation $\left(\lambda=0.75-1.4 \mu \mathrm{m}, 1.4 \mathrm{~W} \mathrm{~cm}^{-2}\right.$, $10 \mathrm{~min})$.

$44.7{ }^{\circ} \mathrm{C}$, respectively. The higher maximum temperature observed for PMNPs and MNPs indicates that $\mathrm{Fe}_{3} \mathrm{O}_{4}$ NPs in PMNPs and MNPs contribute to the NIR-dependent rapid temperature increase and that PMNPs could act as efficient NIR heat absorbers for HTT.

\subsection{In vitro MR/US imaging}

In vitro MRI experiments, PMNPs negatively enhanced the $T_{2}$ weighted MR images (Fig. 4a). Fig. $4 \mathrm{~b}$ shows the $T_{2}$ weighted MRI SI of different groups (I-VI). The $T_{2}$ relaxation rates $\left(1 / T_{2}\right)$ of
PMNPs solutions at different iron concentrations are shown in Fig. 4c. The $T_{2}$ weighted MRI SI decreased with increasing iron concentration, with the transverse relaxivity $\left(r^{2}\right)$ of PMNPs measured as $202.3 \mathrm{mM}^{-1} \mathrm{~S}^{-1}$. The results indicated that the PMNPs produced MR contrast in the transverse photon relaxation-time-weighted sequence.

Before and after NIR irradiation, the brightfield microscopy, B-mode ultrasound and CEUS images of the PMNP and MNP (without PFP) groups were measured (Fig. 5). After NIR irradiation, no phase transition occurred in the PMNP group, and therefore, no gas bubbles were observed under the microscope (Fig. $5 \mathrm{a}_{1}$ ). The nanoparticles exhibited no echo or enhancement in B-mode or CEUS before irradiation (Fig. $5 \mathrm{a}_{2}$ ). After NIR irradiation, when the temperature reached $50{ }^{\circ} \mathrm{C}$, the nanoparticles were gradually converted into microbubbles (Fig. $5 \mathrm{a}_{3}$ ). A significant increase in the ultrasound backscatter signals was observed for the PMNP group (Fig. $5 \mathrm{a}_{4}$ ). Comparatively, no differences were observed under the microscope before and after NIR irradiation for the MNP group (Fig. $5 b_{1}$ and $b_{3}$ ). The Bmode and CEUS images showed no obvious increase in echogenicity or contrast enhancement at $50{ }^{\circ} \mathrm{C}\left(\mathrm{Fig} .5 \mathrm{~b}_{2}\right.$ and $\left.\mathrm{b}_{4}\right)$. The histograms (Fig. 5b and c) showed the echo intensity of the PMNP and MNP groups before and after irradiation in both Bmode and CEUS images. The results showed that microbubble production was consistent with ultrasonography, which is consistent with previous published results. ${ }^{9,25}$

\subsection{In vivo MR/US imaging}

The main objective of our experiments in this study is to determine the feasibility of employing PMNPs as imaging and 
a

a

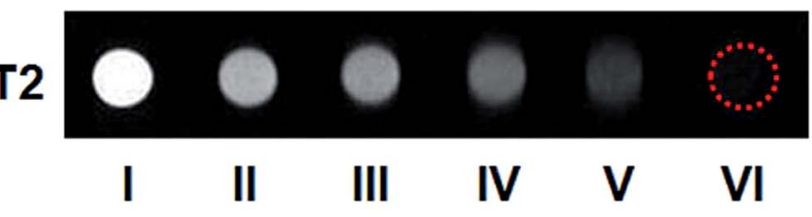

c

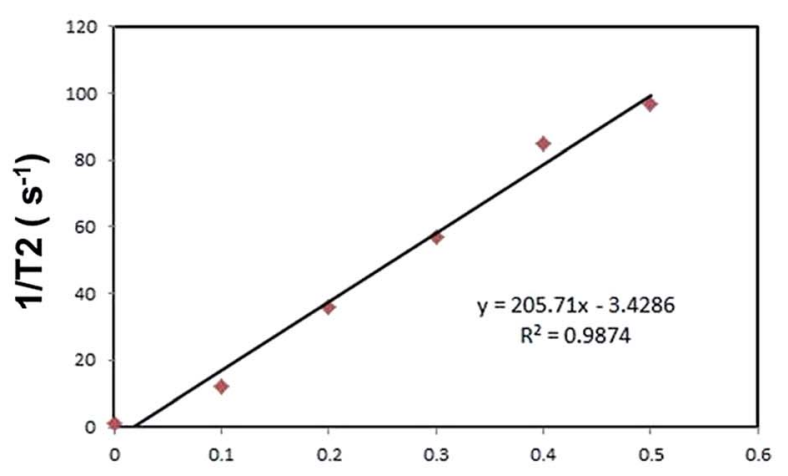

Fe Concentration ( $\mathrm{mM}$ )

\section{b}

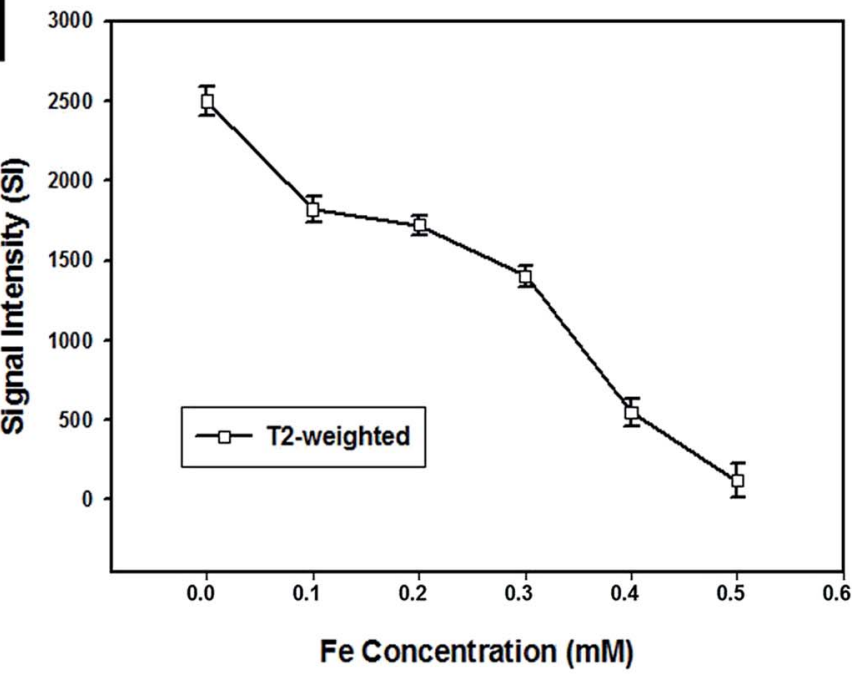

Fig. 4 (a) In vitro $T_{2}$-weighted MR image. PNPs (without iron) (I) and PMNPs with different iron concentrations (II, 0.1 mmol $L^{-1}$; III, 0. $2 \mathrm{mmol} \mathrm{L}^{-1} ; \mathrm{IV}, 0.3 \mathrm{mmol} \mathrm{L}^{-1} ; \mathrm{V}, 0.4 \mathrm{mmol} \mathrm{L}^{-1} ; \mathrm{VI}, 0.5 \mathrm{mmol} \mathrm{L}^{-1}$ ). (b) Different iron concentrations and MR signal intensity of PMNPs. (c) $T_{2}$ relaxation rates $\left(1 / T_{2}\left(s^{-1}\right)\right)$ of PMNP emulsions at different iron concentrations.

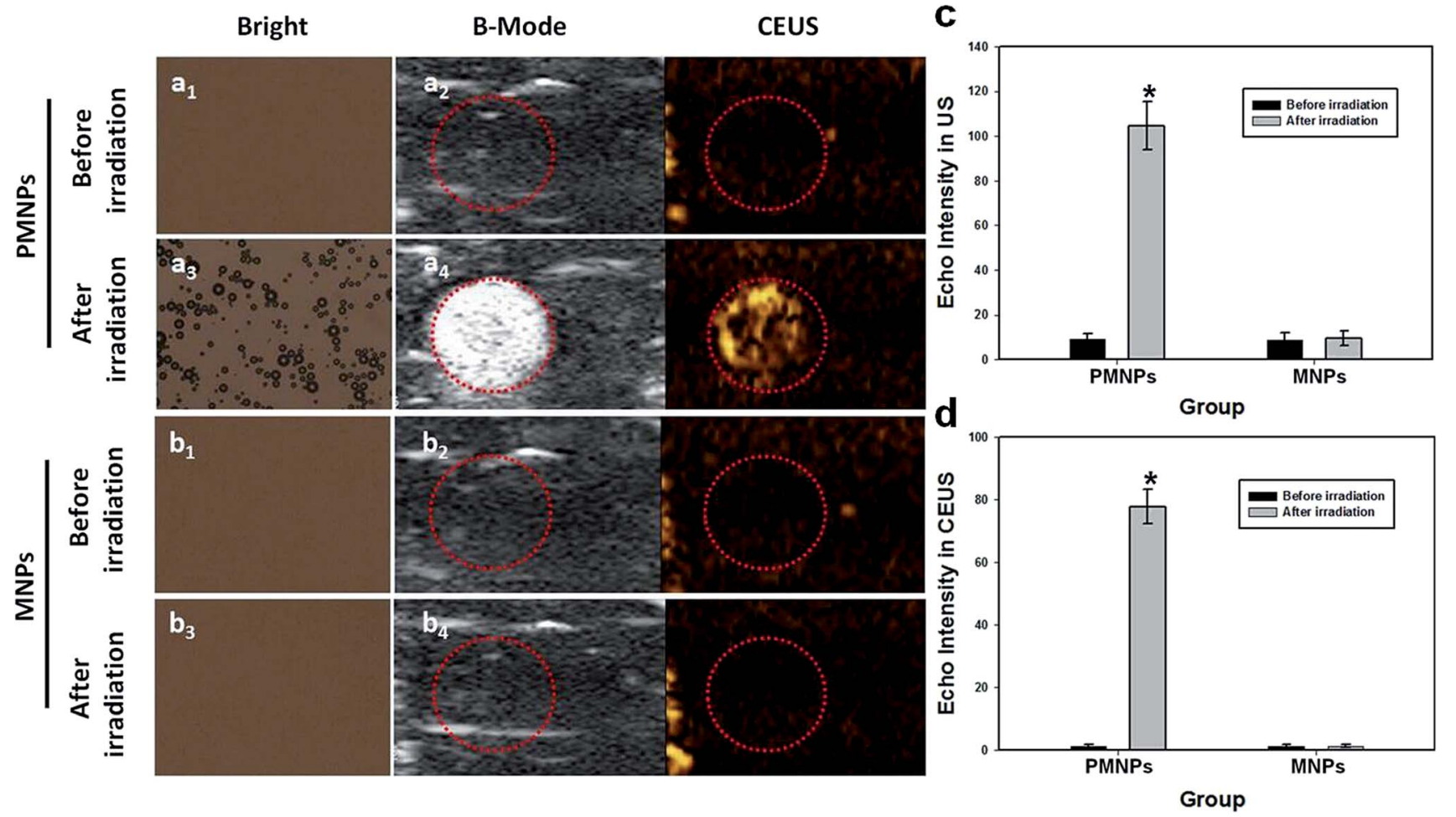

Fig. 5 (a and b) Brightfield microscopy images, ultrasound B-mode and CEUS images of PMNPs (a) and MNPs (without PFP) (b) before and after NIR irradiation $\left(\lambda=0.75-1.4 \mu \mathrm{m}, 1.4 \mathrm{~W} \mathrm{~cm}^{-2}\right)$ in vitro (gel model). $\left(a_{1}-a_{4}\right)$ Magnification, $400 \times$. After NIR irradiation, the nanoparticles gradually convert to microbubbles $\left(a_{3}\right)$. A significant increase in the ultrasound backscatter signal is observed in the PMNP group $\left(a_{4}\right)$. ( $c$ and $\left.d\right)$ Echo intensity of the region of interest before and after NIR irradiation. Echo intensity from the region of interest shown in (b) and using CEUS in (c). The difference is statistically significant $(* p<0.05)$.

synergistic therapeutic agents to strengthen tumor ablation efficiency. Therefore, we used a double dose of nanoparticles in the tumor tissues for imaging and therapy experiments to ensure that all the tumors received the contrast agent, even though a portion of the contrast agent leaked from the tumor to the parenchyma gap. 
Owing to the excellent magnetic and acoustic properties of PMNPs, they were further used as an in vivo dual-mode biological imaging agent. The MR images of tumor tissue pre- and post-injection are shown in Fig. $6 \mathrm{a}$ and b. After percutaneous local injection, $T_{2}$ weighted MR images showed obvious darkening at the tumor site (Fig. 6a and b). The histograms (Fig. 6c) showed that the SI of tumors decreased after PMNP injection. The presence of $\mathrm{Fe}_{3} \mathrm{O}_{4}$ particles in PMNPs showed significantly negative enhancement in tumor tissues. The results demonstrated that PMNPs could be used as an MR imaging contrast agent by shortening the transverse relaxation of surrounding protons, which is consistent with previous work by other investigators. ${ }^{\mathbf{9} 33}$

Next, animal experiments were performed to determine whether ultrasound imaging could be enhanced by NIR irradiation-induced vaporization of PMNPs in vivo. The in vivo US images of tumor tissue pre- and post-injection are shown in Fig. 7. PMNP and MNP groups were imaged before NIR irradiation (Fig. 7a1 and b1) and $10 \mathrm{~s}$ (Fig. 7a2 and b2), $1 \mathrm{~min}$ (Fig. 7a3 and b3) and 4 min (Fig. 7a4 and b4) after irradiation. The red dotted circles indicate the tumor location. Fig. 7a shows an obvious enhancement in ultrasound imaging on Bmode and CEUS after NIR irradiation for $10 \mathrm{~s}$ in the PMNP group (Fig. 7a2); the enhancement was more effective after irradiation for $1 \mathrm{~min}$ and $4 \mathrm{~min}$ (Fig. $7 \mathrm{a} 3$ and a4). However, no enhancement in B-mode and CEUS images was exhibited in MNP group tumors before (Fig. 7b1) and after NIR irradiation for $10 \mathrm{~s}$ (Fig. 7b2), $1 \mathrm{~min}$ (Fig. 7b3), and $4 \mathrm{~min}$ (Fig. 7b4) in both ultrasound modes. Fig. 7c shows the temperature changes in tumor tissues in the PMNP and MNP groups. The tumor tissue temperature increased with the irradiation time increase in both groups. The gray value changes in the tumor were detected in both B-mode and CEUS, the contrast enhancement was reported in Fig. 7d and e. A significant difference was observed between pre- and post-irradiation gray values in both B-mode and CEUS, at all three time points in the PMNP group $\left({ }^{*} p<0.05\right)$. The results showed that the PMNPs absorbed the NIR light and subsequently converted it to heat, which increased the internal temperature above the boiling point of the liquid, inducing a liquid-gas phase shift and generating lots of microbubbles. This microbubble generation further enhanced B-mode and CEUS ultrasound imaging in tumor tissues. The MNPs exhibited no liquid-gas phase changes and therefore could not generate microbubbles. Thus, the ultrasound image was not enhanced. The results showed that the elaborately designed PMNPs can be transformed from liquid into gas by infrared radiation, thereby facilitating the thermoelastic expansion of the inner liquid PFP and enhancing the thermal ablation effect on local tumors.

Therefore, the integrated PMNPs provided three imaging modalities with a high resolution MR probe for deep tissues and an ultrasound probe for superficial organs that can be used for a variety of diseases. The presence of $\mathrm{Fe}_{3} \mathrm{O}_{4}$ and the microbubbles generated by the phase-transition process conferred imaging properties that render the PMNPs ideal for use in MR/ US imaging-guided tumor therapy. Moreover, combination of dual-modal detectability in the same agent could reduce the burden of liver and kidney metabolism on patients for many kinds of contrast agents for different examinations.

\subsection{HTT for tumor ablation in vivo}

The temperature in tumor regions in groups I-IV (I, saline; II, PMNPs; III, PLGA + NIR; IV, PMNPs + NIR) were monitored with a thermocouple thermometer during NIR irradiation. The tumor temperature rapidly rose to $45.0^{\circ} \mathrm{C}$ and continued to increase to $55.0^{\circ} \mathrm{C}$ after $4 \mathrm{~min}$ of NIR irradiation in mice who received PMNPs (group IV). However, the tumor temperature in
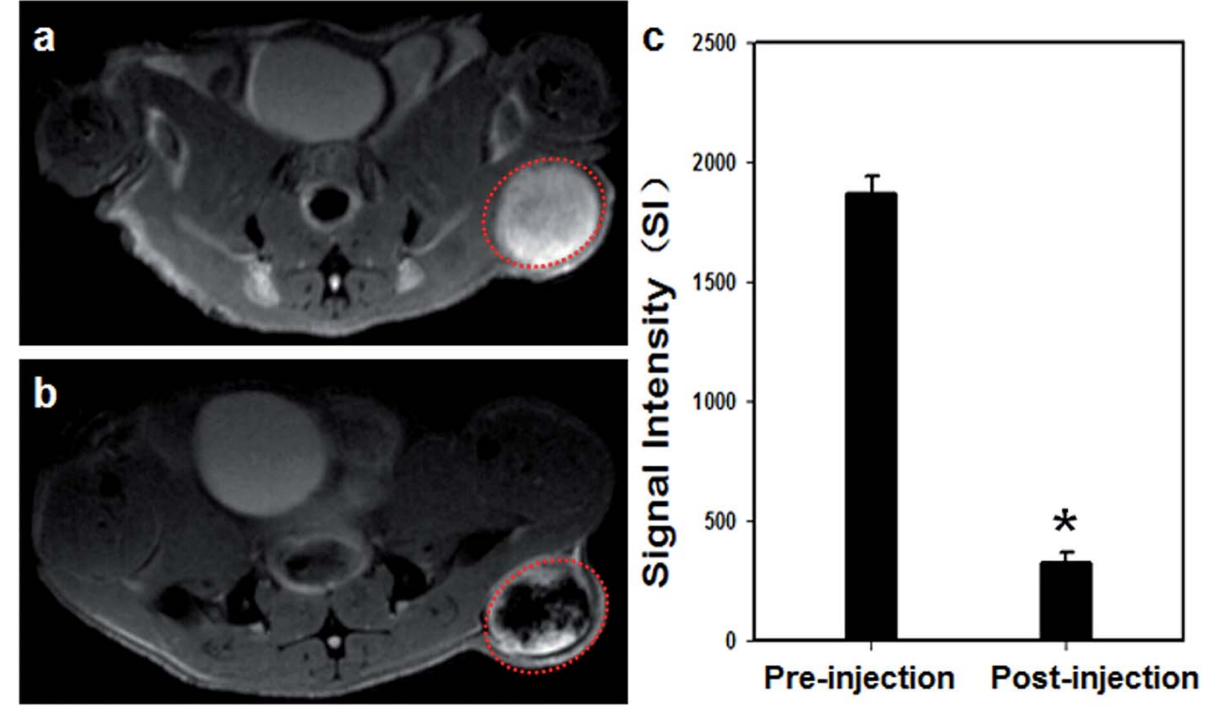

Fig. 6 (a and b) In vivo tumor $T_{2}$-weighted MR image of nude mice (tumor areas in red circle) before and after percutaneous local injection of PMNPs. (c) Signal intensity of $T_{2}$-weighted MR after injection shows significant negative contrast enhancement in tumor tissues. The difference is statistically significant $(* p<0.05)$. 
mice who received PLGA (group III) showed a little increase upon NIR irradiation for the same duration. The tumor temperature in mice treated with saline (group I) or pure PMNPs without NIR irradiation (group II) did not significantly increase
(Fig. 8a). Then, the body weights and tumor sizes of different groups were monitored every 2 days after treatments. As shown in Fig. 8b, no obvious body weight dropping and apparent differences among each group during the treatment were
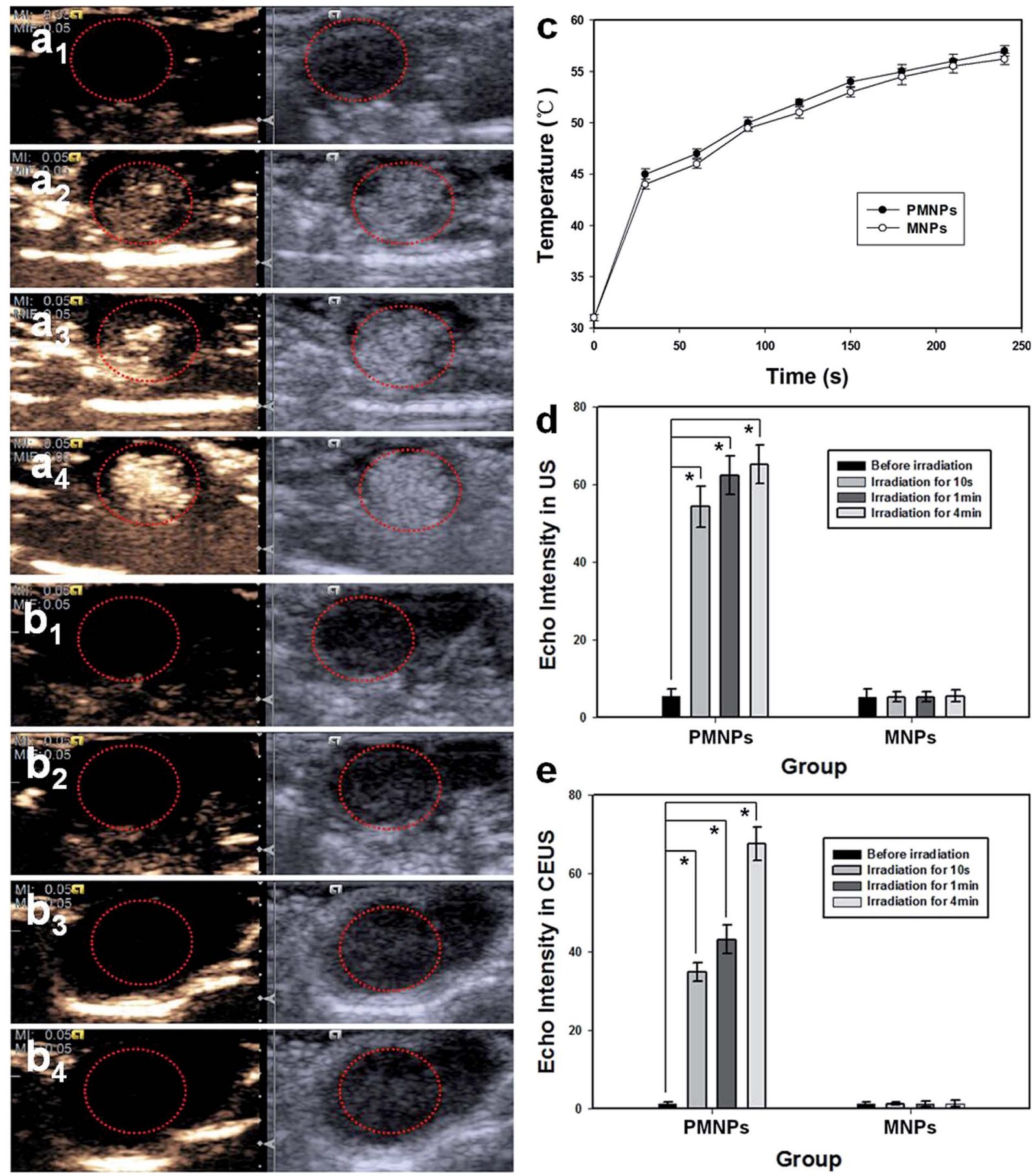

Fig. 7 (a and b) In vivo ultrasound B-mode and CEUS images in mouse tumors before and after NIR irradiation $\left(\lambda=0.75-1.4 \mu \mathrm{m}, 1.4 \mathrm{~W} \mathrm{~cm}^{-2}\right)$ for PMNPs (a) and MNPs (without PFP) (b). Two ultrasound images (CEUS (left) and B-mode (right)) of the PMNP and MNP groups before irradiation $\left(a_{1}\right.$ and $\left.b_{1}\right)$ and after irradiation for $10 \mathrm{~s}\left(a_{2}\right.$ and $\left.b_{2}\right), 1 \mathrm{~min}\left(a_{3}\right.$ and $\left.b_{3}\right)$ and $4 \mathrm{~min}\left(a_{4}\right.$ and $\left.b_{4}\right)$. The temperature changes in mouse tumor tissues in PMNP and MNP groups at a distance of $15 \mathrm{~cm}$ for $4 \mathrm{~min}$ (c). The red dotted circles indicate tumor location. The PMNP group shows a significant increase in tumor both in B-mode and CEUS after NIR irradiation ( $d$ and e). Echo intensity of the region of interest before and after NIR irradiation. Echo intensity from the region of interest shown in (d) and using CEUS in (e). The difference is statistically significant $(* p<0.05)$. 

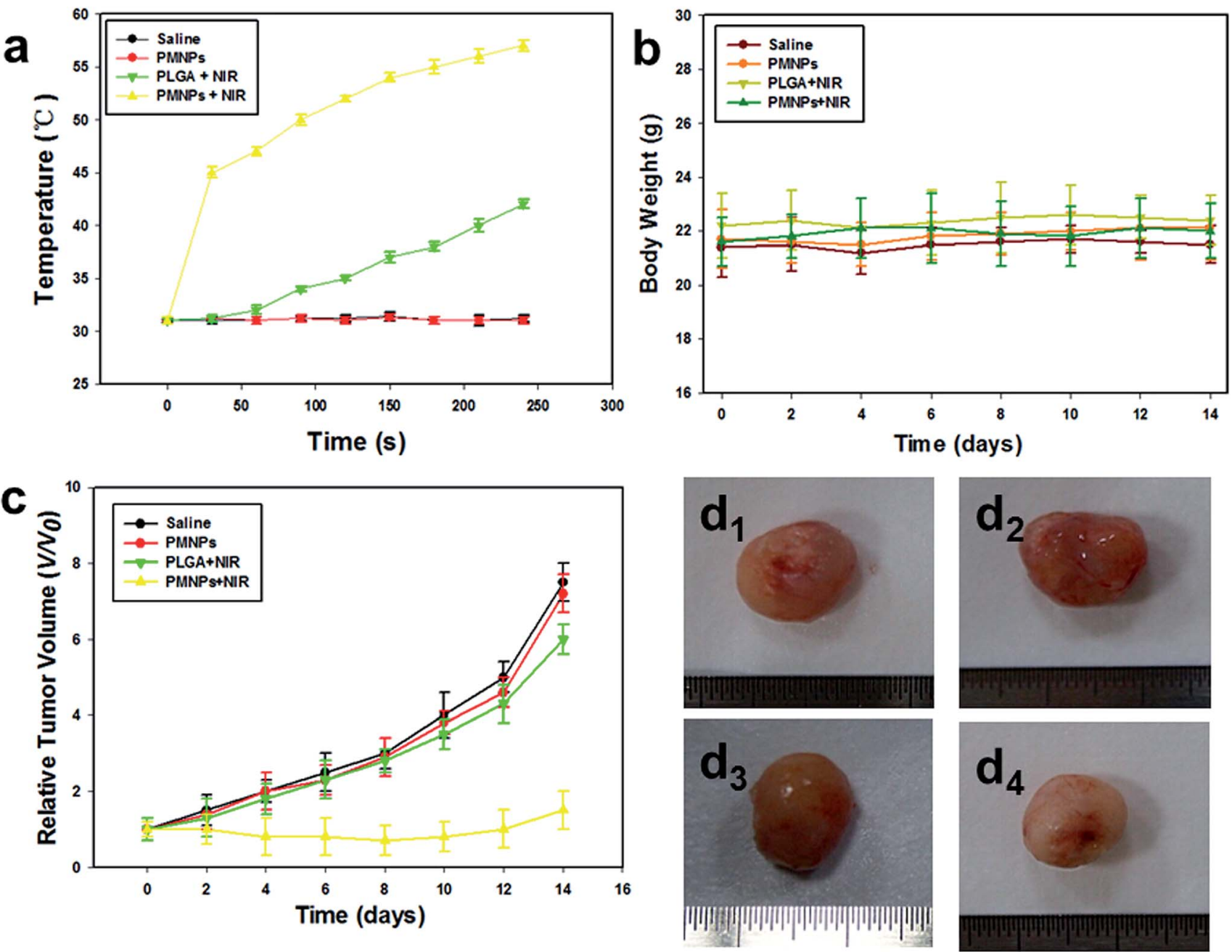

Fig. 8 (a) Temperature increased with extended irradiation time ( $\left.\lambda=0.75-1.4 \mu \mathrm{m}, 1.4 \mathrm{~W} \mathrm{~cm}{ }^{-2}, 4 \mathrm{~min}\right)$ in groups I-IV (I, saline; II, PMNPs; III, PLGA + NIR; IV, PMNPs + NIR). (b) Body weight curves of tumor-bearing mice in groups I-IV recorded during 14 day evaluation period. (c) Tumor growth curves of tumor-bearing mice in groups I-IV indicated during the treatment progress. (d) Digital pictures of macroscopic tumor tissues in groups I-IV.

observed. Fig. 8c shows the tumor growth rate was significantly inhibited in group IV. In contrast, in the groups I-III, the volumes of tumors increased rapidly by $6-8$ fold during the treatment.

Fig. 8d shows photographs of the excised tumor tissues from the mice in group I-IV. There was no obvious change in color between groups I-III (Fig. $8 \mathrm{~d}_{1-3}$ ). Comparatively, the color of tumors in group IV was much whiter than that of groups I-III. From examination with the naked eye, group IV appeared to have a greater synergistic effect on tumor ablation.

On microscopic examination of HE-stained sections, the tumor tissues excised from group I-III mice showed no distinct signs of necrosis, and the tumor cells showed intact arrangement in nests (Fig. 9a-c). In group IV, lots of microbubbles were observed on the HE-stained tumor tissues, which further confirmed the liquid-gas phase shift (Fig. 9d). Severe cell injury with lysed nuclear material and cell membranes and vacuole formation was observed. Typical signs of cell injury between necrotic and non-necrotic regions with a sharp demarcation were seen with contrast agent injection in group IV, which demonstrates a high degree of damage to tumor cells. These findings indicate a more efficient synergistic effect for HTT ablation.

In all groups, necrosis was more evident in the contrast agent injection region of the excised tumor specimens, which is consistent with the findings of HE-stained sections on TEM (Fig. 10). In group I (saline), the cell and nuclear membranes were intact, and no particles were observed in tumor tissue (Fig. 10a1 and a2). In group II (PMNPs), the tissue architecture was retained, and lots of nanoparticles were found in the tumor tissue; the iron particles were clearly visible in the PMNP shells (Fig. 10b1 and b2). In group III (PLGA + NIR), the cell structures were normal and clear, and lots of nanoparticles without iron particles were found in the tumor tissue (Fig. 10c1 and c2). In group IV (PMNPs + NIR), some cell membranes were ruptured, the nuclear membrane showed 

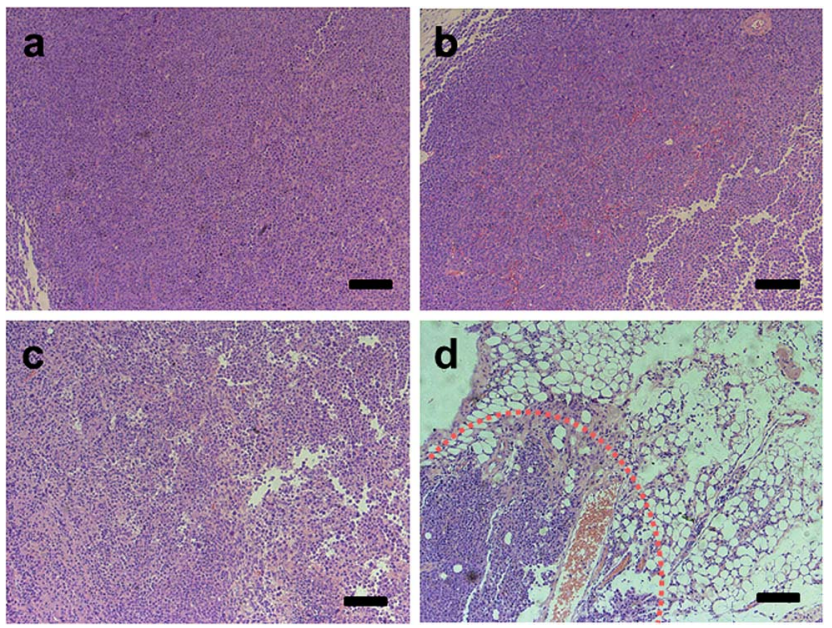

Fig. 9 Pathological examination results of nude tumor tissues in groups I-IV (I, saline; II, PMNPs; III, PLGA + NIR; IV, PMNPs + NIR). (ad) Magnification, 100x; scale bar, $100 \mu \mathrm{m}$. In group IV, a large number of small bubbles are observed in the tumor tissues after HE staining, severe cell damage is seen, with fragmentation of lysed cell and nuclear membranes, leaving a mass of vacuoles (d). The red dotted curve indicates typical damage with a sharp demarcation between necrotic and non-necrotic regions in the contrast agent injection region.

disintegration, and many microbubbles were generated with iron particles expelled to the edge or outside of the microbubbles (Fig. 10d1 and d2).
The mechanism of the synergistic effect of PMNPs in HTT ablation might involve the following events. On the one hand, $\mathrm{Fe}_{3} \mathrm{O}_{4}$ nanoparticles present in PFMNPs absorbed the NIR light and induced heat in tissue. PFP acted as a phase change agent and generated microbubbles in situ upon exposure to NIR irradiation, which enhanced the cavitation and tumor ablation effect. The NIR-induced droplet vaporization mechanism enhanced the ultrasound imaging and therapy capabilities of the nanoparticles. Therefore, the nanoparticles are amenable to selective vaporization and able to generate sufficient microbubbles within an ROI to destroy tumor tissues.

\subsection{In vivo toxicity of PMNPs}

To further assess the in vivo toxicity of PMNPs, major organs (lung, heart, liver, spleen, and kidney) of healthy BABL/c mice intravenously injected with PMNPs $\left(0.2 \mathrm{~mL}, 2 \mathrm{mg} \mathrm{mL}^{-1}\right)$ or saline were harvested for histological analysis after 14 days. In addition, blood samples of these mice were collected at 3 and 14 days after injection of PMNPs or saline. No noticeable organ damage was observed in H\&E stained sections of major organs in two groups (Fig. 11a). As shown in Fig. 11b, the levels of these four indicators (ALT, AST, BUN and CREA) were within normal ranges with no significant differences between the different time points (all $p>0.05$ ). Thus, the histological examination and serum biochemistry results indicate that PMNPs did not induce appreciable toxic side effects in treated animals.
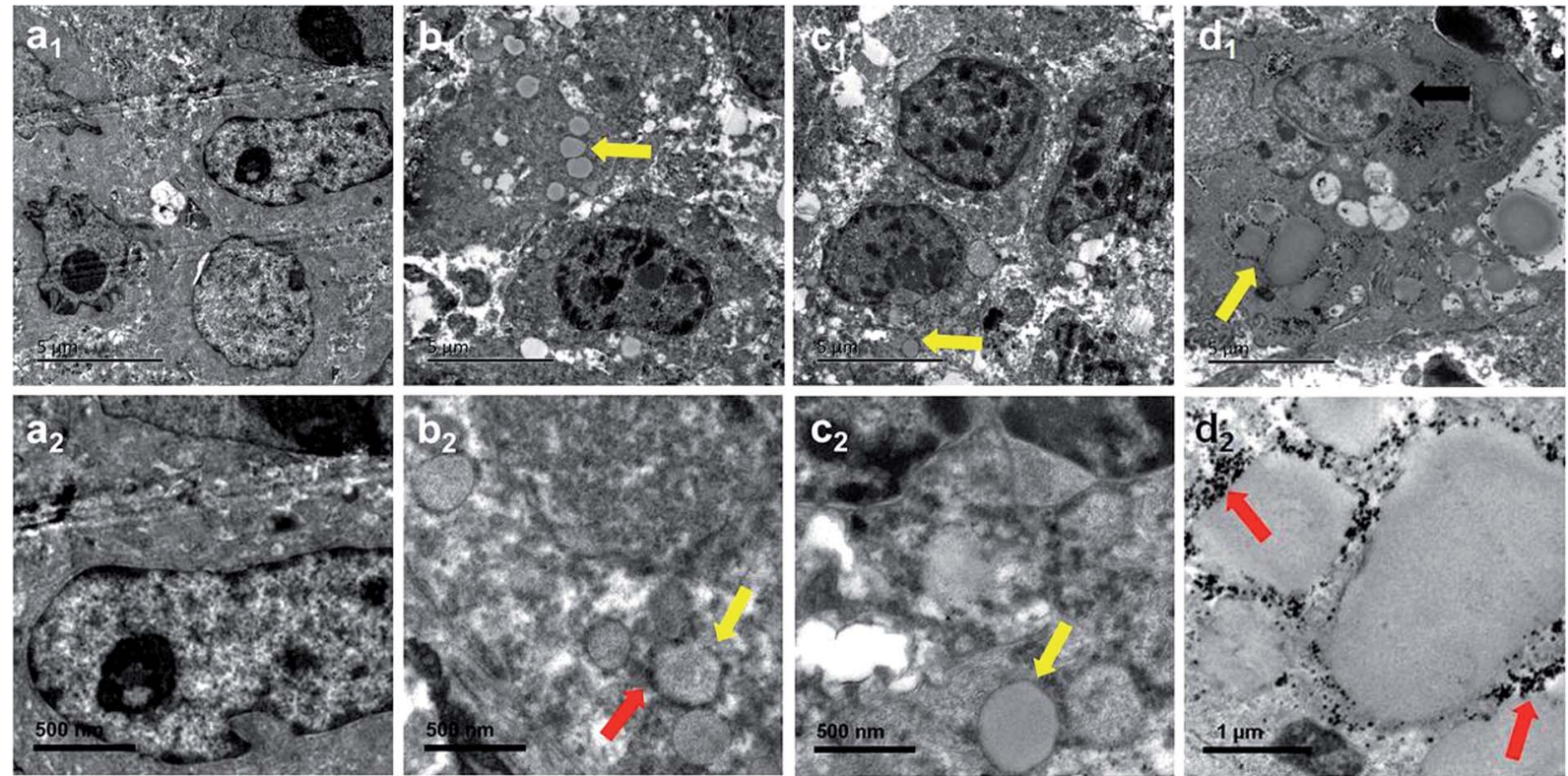

Fig. 10 TEM examination results of mouse tumor tissue in groups I-IV (I, saline; II, PMNPs; III, PLGA + NIR; IV, PMNPs + NIR). ( $a_{1}$ and $\left.d_{1}\right)$ Magnification, 3900x; scale bar, $5 \mu \mathrm{m}$. ( $\mathrm{a}_{2}$ and $\mathrm{c}_{2}$ ) Magnification, $37000 \times$; scale bar, $500 \mathrm{~nm}$. (d $\left.\mathrm{d}_{2}\right)$ Magnification, $18500 \times$; scale bar, $1 \mu \mathrm{m}$. As shown in (b and d), many nanoparticles (yellow arrows) appear in the tumor tissues. A number of $\mathrm{Fe}_{3} \mathrm{O}_{4}$ nanoparticles are embedded in the spherical shell in the form of black particles shown in $(b-d)$; generation of many microbubbles shown in (d); black arrows indicate the interrupted cell membranes and nuclear membranes. In group IV, some cell membranes are ruptured, nuclear membranes are disintegrated, and lots of microbubbles generated with iron particles are expelled to the edge or outside of the microbubbles $\left(d_{1}\right.$ and $\left.d_{2}\right)$. 
a

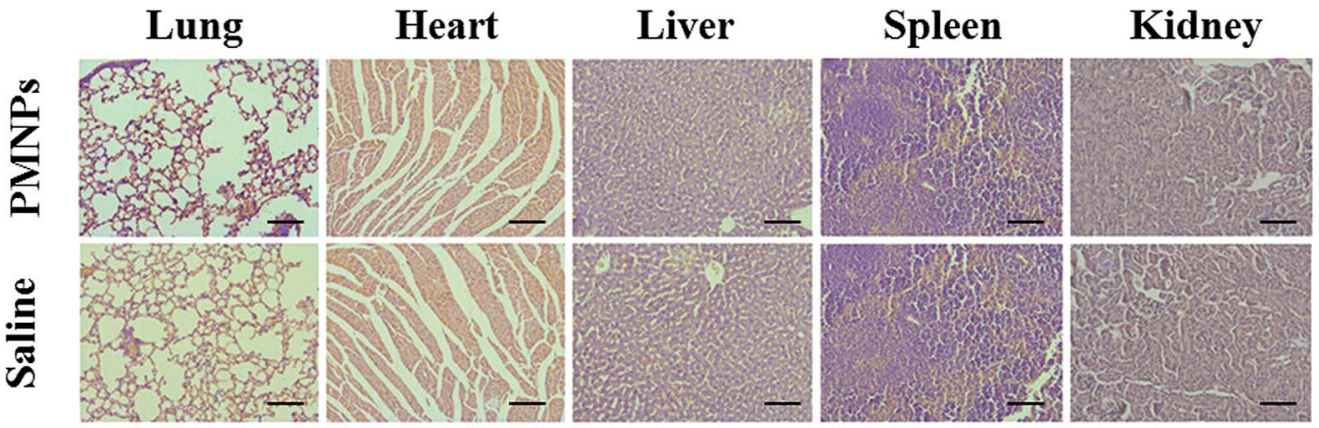

b Serum biochemical indicators after the injection of PMNPs（2 mg/mL）

\begin{tabular}{|l|c|c|c|}
\hline & Pre-injection & 3d & 14d \\
\hline ALT (U/L) & $39.85 \pm 15.77$ & $44.17 \pm 8.66$ & $42.15 \pm 2.08$ \\
\hline AST (U/L) & $109.95 \pm 16.78$ & $122.46 \pm 26.84$ & $113.06 \pm 14.34$ \\
\hline BUN (mM) & $7.21 \pm 0.89$ & $8.90 \pm 1.27$ & $7.61 \pm 1.32$ \\
\hline CREA ( $\mu M$ ) & $7.28 \pm 1.31$ & $9.57 \pm 1.50$ & $8.52 \pm 2.51$ \\
\hline
\end{tabular}

Note: The same indicators at different time points for pairwise comparisons ( $P>0.05$ for all of the comparisons).

Fig. 11 In vivo toxicity study. (a) H\&E stained sections of major organs (lung, heart, liver, spleen, and kidney) harvested 14 days after intravenous injection of PMNPs $\left(0.2 \mathrm{~mL}, 2 \mathrm{mg} \mathrm{mL}^{-1}\right)$ or saline. No noticeable abnormality was observed in two groups. (b) Levels of serum biochemical markers (ALT, AST, BUN and CREA) before and after injection of PMNPs or saline at 3 and 14 days.

\section{Conclusion}

The synthesized multifunctional PMNPs used in this study show promise as a theranostic agent for cancer dual-modal imaging and hyperthermia treatment. The dual-modal contrast agent $\mathrm{Fe}_{3} \mathrm{O}_{4}$ nanoparticles were embedded in the shell, and the microbubbles generated using the liquid-gas phase shift process conferred MR/US imaging properties to PMNPs, thus enabling imaging-guided tumor therapy. This successful demonstration of the use of nanobiotechnology for stimuli-responsive MR/US imaging and NIR-induced HTT therapy provides an alternative modality for strong "nanotheranostics". The progress of $\mathrm{Fe}_{3} \mathrm{O}_{4}$ nanoparticles release into a desired region after vaporization, may be useful for the understanding of drug release from an inflated bubble, thereby achieving a high concentration dose at target tissue and minimizing systemic side effects.

\section{Conflicts of interest}

The authors declare that they have no conflicts of interest. Animals were performed in accordance with the guidelines of the Department of Laboratory Animals, Central South University, China, and approved by the Ethics Committee of Central South University. Informed consent was obtained from all human participants included in the study.

\section{Acknowledgements}

The authors thank Zhigang Wang and Pan Li (Chongqing Medical University) for their technical assistance. This project was funded by the National Natural Science Foundation of China (Grant No. 81401431 and 81601883), and the Hunan Provincial Natural Science Foundation of China (Grant No. 2017JJ3443).

\section{References}

1 K. L. Donaldson, G. L. Goolsby and A. F. Wahl, Cytotoxicity of the anticancer agents cisplatin and taxol during cell proliferation and the cell cycle, Int. J. Cancer, 1994, 57, 847-855.

2 Q. Chen, X. Wang, C. Wang, L. Feng, Y. Li and Z. Liu, DrugInduced Self-Assembly of Modified Albumins as Nanotheranostics for Tumor-Targeted Combination Therapy, ACS Nano, 2015, 9, 5223-5233.

3 X. Song, R. Zhang, C. Liang, Q. Chen, H. Gong and Z. Liu, Nano-assemblies of J-aggregates based on a NIR dye as a multifunctional drug carrier for combination cancer therapy, Biomaterials, 2015, 57, 84-92.

4 K. Hayashi, M. Nakamura, H. Miki, S. Ozaki, M. Abe, T. Matsumoto, et al., Magnetically responsive smart nanoparticles for cancer treatment with a combination of magnetic hyperthermia and remote-control drug release, Theranostics, 2014, 4, 834-844. 
5 C. He, X. Duan, N. Guo, C. Chan, C. Poon, R. R. Weichselbaum, et al., Core-shell nanoscale coordination polymers combine chemotherapy and photodynamic therapy to potentiate checkpoint blockade cancer immunotherapy, Nat. Commun., 2016, 7, 12499.

6 L. E. Aguilar, A. GhavamiNejad, C. H. Park and C. S. Kim, Ondemand drug release and hyperthermia therapy applications of thermoresponsive poly-(NIPAAm-co-HMAAm)/polyurethane core-shell nanofiber mat on non-vascular nitinol stents, Nanomedicine, 2017, 13, 527-538.

7 A. Gladwish, M. Milosevic, A. Fyles, J. Xie, J. Halankar, U. Metser, et al., Association of Apparent Diffusion Coefficient with Disease Recurrence in Patients with Locally Advanced Cervical Cancer Treated with Radical Chemotherapy and Radiation Therapy, Radiology, 2016, 279, 158-166.

8 J. Huang, J. S. Xu and R. X. Xu, Heat-sensitive microbubbles for intraoperative assessment of cancer ablation margins, Biomaterials, 2010, 31, 1278-1286.

9 Y. Zhao, W. Song, D. Wang, H. Ran, R. Wang, Y. Yao, et al., Phase-Shifted PFH@PLGA/ $\mathrm{Fe}_{3} \mathrm{O}_{4}$ Nanocapsules for MRI/US Imaging and Photothermal Therapy with near-Infrared Irradiation, ACS Appl. Mater. Interfaces, 2015, 7, 1423114242.

10 D. B. Brown, Concepts, considerations, and concerns on the cutting edge of radiofrequency ablation, J. Vasc. Intervent. Radiol., 2005, 16, 597-613.

11 W. J. Lokerse, M. Bolkestein, T. L. Ten Hagen, M. de Jong, A. M. Eggermont, H. Grull, et al., Investigation of Particle Accumulation, Chemosensitivity and Thermosensitivity for Effective Solid Tumor Therapy Using Thermosensitive Liposomes and Hyperthermia, Theranostics, 2016, 6, 17171731.

12 T. Lammers, F. Kiessling, W. E. Hennink and G. Storm, Nanotheranostics and image-guided drug delivery: current concepts and future directions, Mol. Pharm., 2010, 7, 18991912.

13 S. Mura and P. Couvreur, Nanotheranostics for personalized medicine, Adv. Drug Delivery Rev., 2012, 64, 1394-1416.

14 F. M. Kievit and M. Zhang, Cancer nanotheranostics: improving imaging and therapy by targeted delivery across biological barriers, Adv. Mater., 2011, 23, H217-H247.

15 P. Zhang, C. Hu, W. Ran, J. Meng, Q. Yin and Y. Li, Recent Progress in Light-Triggered Nanotheranostics for Cancer Treatment, Theranostics, 2016, 6, 948-968.

16 D. Park, K. O. Ahn, K. C. Jeong and Y. Choi, Polypyrrolebased nanotheranostics for activatable fluorescence imaging and chemo/photothermal dual therapy of triplenegative breast cancer, Nanotechnology, 2016, 27, 185102.

17 L. Wang, Y. Hu, Q. Peng, J. Zhou, Q. Zhou, S. An, et al., Indocyanine-green-loaded microbubbles for localization of sentinel lymph node using near-infrared fluorescence/ ultrasound imaging: a feasibility study, RSC Adv., 2016, 6, 50513-50520.

18 C. Niu, Z. Wang, G. Lu, T. M. Krupka, Y. Sun, Y. You, et al., Doxorubicin loaded superparamagnetic PLGA-iron oxide multifunctional microbubbles for dual-mode US/MR imaging and therapy of metastasis in lymph nodes, Biomaterials, 2013, 34, 2307-2317.

19 C. Niu, Z. Wang, G. Zuo, T. M. Krupka, H. Ran, P. Zhang, et al., Poly(Lactide-co-glycolide) ultrasonographic microbubbles carrying Sudan black for preoperative and intraoperative localization of lymph nodes, Clin. Breast Cancer, 2012, 12, 199-206.

20 A. Espinosa, R. Di Corato, J. Kolosnjaj-Tabi, P. Flaud, T. Pellegrino and C. Wilhelm, Duality of Iron Oxide Nanoparticles in Cancer Therapy: Amplification of Heating Efficiency by Magnetic Hyperthermia and Photothermal Bimodal Treatment, ACS Nano, 2016, 10, 2436-2446.

21 K. Hayashi, M. Nakamura, W. Sakamoto, T. Yogo, H. Miki, S. Ozaki, et al., Superparamagnetic nanoparticle clusters for cancer theranostics combining magnetic resonance imaging and hyperthermia treatment, Theranostics, 2013, 3, 366-376.

22 D. Kripfgans, J. B. Fowlkes, D. L. Miller, O. P. Eldevik and P. L. Carson, Acoustic droplet vaporization for therapeutic and diagnostic applications, Ultrasound. Med. Biol., 2000, 26, 1177-1189.

23 N. Reznik, R. Williams and P. N. Burns, Investigation of vaporized submicron perfluorocarbon droplets as an ultrasound contrast agent, Ultrasound. Med. Biol., 2011, 37, 1271-1279.

24 Y. Sun, Y. Wang, C. Niu, E. M. Strohm, Y. Zheng, H. Ran, et al., Laser-Activatible PLGA Microparticles for ImageGuided Cancer Therapy In Vivo, Adv. Funct. Mater., 2014, 24, 7674-7680.

25 Y. Zhou, Z. Wang, Y. Chen, H. Shen, Z. Luo, A. Li, et al., Microbubbles from gas-generating perfluorohexane nanoemulsions for targeted temperature-sensitive ultrasonography and synergistic HIFU ablation of tumors, Adv. Mater., 2013, 25, 4123-4130.

26 M. Zhang, M. Fabiilli, P. Carson, F. Padilla, S. Swanson, O. Kripfgans, et al., Acoustic Droplet Vaporization for the Enhancement of Ultrasound Thermal Therapy, Proc. - IEEE Ultrason. Symp., 2010, 2010, 221-224.

27 M. Zhang, M. L. Fabiilli, K. J. Haworth, F. Padilla, S. D. Swanson, O. D. Kripfgans, et al., Acoustic droplet vaporization for enhancement of thermal ablation by high intensity focused ultrasound, Acad. Radiol., 2011, 18, 11231132.

28 N. Y. Rapoport, A. M. Kennedy, J. E. Shea, C. L. Scaife and K. H. Nam, Controlled and targeted tumor chemotherapy by ultrasoundactivated nanoemulsions/microbubbles, J. Controlled Release, 2009, 138, 268-276.

29 M. L. Fabiilli, K. J. Haworth, I. E. Sebastian, O. D. Kripfgans, P. L. Carson and J. B. Fowlkes, Delivery of chlorambucil using an acoustically-triggered perfluoropentane emulsion, Ultrasound. Med. Biol., 2010, 36, 1364-1375.

30 N. Rapoport, K. H. Nam, R. Gupta, Z. Gao, P. Mohan, A. Payne, et al., Ultrasound-mediated tumor imaging and nanotherapy using drug loaded, block copolymer stabilized perfluorocarbon nanoemulsions, J. Controlled Release, 2011, 153, 4-15. 
31 C. Niu, L. Wang, Z. Wang, Y. Xu, Y. Hu and Q. Peng, Laser irradiated fluorescent perfluorocarbon microparticles in 2D and 3-D breast cancer cell models, Sci. Rep., 2017, 7, 43408.

32 C. Niu, Y. Xu, S. An, M. Zhang, Y. Hu, L. Wang, et al., Nearinfrared induced phase-shifted ICG/ $\mathrm{Fe}_{3} \mathrm{O}_{4}$ loaded PLGA nanoparticles for photothermal tumor ablation, Sci. Rep., 2017, 7(1), 5490.

33 Y. Sun, Y. Zheng, H. Ran, Y. Zhou, H. Shen, Y. Chen, et al., Superparamagnetic PLGA-iron oxide microcapsules for dual-modality US/MR imaging and high intensity focused US breast cancer ablation, Biomaterials, 2012, 33, 5854-5864. 